\title{
The Role of Steatosis in HBsAg Seroclearance for Patients with Chronic Hepatitis B Infection: Fact or Fiction?
}

\author{
James Fung $\cdot$ Man-Fung Yuen $\cdot$ Ching-Lung Lai
}

Published online: 27 October 2012

(C) The Author(s) 2012. This article is published with open access at Springerlink.com

In the Asia Pacific region, chronic hepatitis $\mathrm{B}(\mathrm{CHB})$ is an endemic infection, and is the most important cause of cirrhosis and hepatocellular carcinoma (HCC). The understanding of the natural history of $\mathrm{CHB}$ through large longitudinal population studies has been pivotal in identifying risk factors which are important for disease progression $[1,2]$. Well-established risk factors include host factors, such as age and gender, and viral factors, such as genotype and viral load. The interplay between other types of liver diseases and CHB is less well studied. Non-alcoholic fatty liver disease (NAFLD), an entity which has traditionally been associated with Western populations, is now becoming more prevalent in many parts of Asia. This is partly due to the rising prevalence of obesity in the Asia Pacific countries. Therefore, it is unavoidable that there will be an increasing trend of NAFLD co-existing with other liver diseases. The effects of NAFLD on viral hepatitis have been described mainly in patients with chronic hepatitis $\mathrm{C}$. In this setting, the presence of steatosis can potentially accelerate fibrogenesis, increase the risk of cirrhosis and hepatocellular carcinoma (HCC), and reduce the efficacy of antiviral therapy [3].

In contrast to chronic hepatitis $\mathrm{C}$, the data regarding the effect of steatosis on CHB, and vice versa, is sparse. This is in spite of the fact that an increasing proportion of CHB patients have evidence of steatosis. There are currently no human studies implicating hepatitis B virus (HBV) to be steatogenic. To date, only studies using transgenic mice have

J. Fung · M.-F. Yuen · C.-L. Lai ( $₫)$

Department of Medicine, The University of Hong Kong, Hong

Kong, Hong Kong, SAR

e-mail: hrmelcl@hku.hk

J. Fung · M.-F. Yuen · C.-L. Lai

State Key Laboratory for Liver Research, The University of

Hong Kong, Hong Kong, Hong Kong, SAR demonstrated the ability of HBV protein $X$ to induce the expression of lipid synthesis genes [4]. In humans, steatosis appears to be mediated predominantly by host demographic and anthropometric factors and the presence of metabolic factors such as diabetes mellitus, hypertension, and dyslipidemia. A recent study by Wong et al. [5] on 91 CHB patients and 922 controls showed that HBV infection was independently associated with a lower rate of steatosis after adjusting for demographic and metabolic factors (adjusted OR 0.42, $95 \%$ CI $0.20-0.88$ ). Therefore, in humans, HBV does not appear to be steatogenic and, in fact, may have the opposite effect.

The role of steatosis as an independent factor on CHB outcome is also not well defined. Large population cohorts have shown that the presence of obesity and metabolic syndrome are associated with increased risk for liver cirrhosis and HCC in patients with CHB. A study of 2,903 male CHB patients with a mean follow-up of 14.7 years identified excess body weight as a significant risk for HCC development and liver-related mortality [6]. Chen et al. [7] demonstrated a higher risk for developing HCC in 3,931 CHB patients, with diabetes being a significant association. A study of 1,566 CHB patients by Wong et al. [8] showed an increased risk of cirrhosis for those with underlying metabolic syndrome. Although metabolic factors appear to be risk factors for the development of cirrhosis and HCC in $\mathrm{CHB}$, the role of steatosis as an independent risk factor is not conclusive. The lack of association between steatosis and fibrosis/cirrhosis in CHB has been shown in several studies. Furthermore, a seemingly paradoxical relationship has been observed in studies showing a potential protective role of steatosis in reducing the severity of liver disease in CHB. A meta-analysis of 7 studies comprising 3,067 patients shows a negative effect of steatosis on viral load, with a standardized mean difference of -74.12 (95\% CI, 
-82.93 to -65.31 ) [9]. Shi et al. [10] reported a significantly lower rate of $F \geq 2$ fibrosis in $260 \mathrm{CHB}$ patients with steatosis compared to 1,655 without steatosis (39.6 vs. $53.5 \%$, respectively, $P<0.05)$. Another study of 86 males with $\mathrm{CHB}$ showed no significant association between significant fibrosis and the presence of steatosis [11]. Likewise, a recent study of $174 \mathrm{CHB}$ patients showed no significant difference in fibrosis or necroinflammation between those with and without steatosis [12].

Although the effect of steatosis on the severity of liver disease in $\mathrm{CHB}$ continues to be uncertain, even less is known about its role in the natural history of HBV infection. The transition from the immune-tolerant phase to the immuneclearance phase and to subsequent $\mathrm{HBeAg}$ seroconversion, and occasionally to $\mathrm{HBsAg}$ seroclearance, is largely immune-mediated. Given the lack of pathophysiological linkage between steatosis and HBV, the role of steatosis is likely to be minimal in these instances. In a previous study from 2007, Chu et al. [13] performed a case-control study looking at $54 \mathrm{HBsAg}$ carriers with evidence of HBsAg seroclearance and compared it with 108 carriers without HBsAg seroclearance matched for age and sex. The study reported a higher percentage of moderate-severe steatosis in those with HBsAg seroclearance, and concluded that steatosis may have contributed to the loss of HBsAg. The mechanisms for this observed phenomenon is unclear, although the authors surmise that the presence of fat within the hepatocytes may alter HBsAg cytoplasmic distribution and lead to subsequent seroclearance. A further proposed mechanism postulated that steatosis may induce apoptosis which may in turn contribute to the loss of HBsAg. However, it is unlikely that any firm conclusion can actually be drawn from this initial study. The quality and the selection criteria of the control subjects are critical in any case-control studies. In this particular study, it is insufficient to match only for age and gender without the consideration of important viral parameters such as genotype and viral load. Also, no information was given as to whether the controls were consecutive patients who fulfilled their matching criteria. A multivariate analysis is also needed to determine whether in fact steatosis itself or other factors such as obesity or metabolic risk factors were more important in causing HBsAg seroclearance.

In this issue of Digestive Diseases and Sciences, Chu et al. [14] performed a follow-up study looking only at the patients who achieved HBsAg seroclearance. Of the 155 patients studied, they observed that those with hepatic steatosis achieved HBsAg at a younger age compared to those without steatosis (49 vs. 53 years, respectively, $P=0.001)$. One can assume that a third of the patients of this study were in fact from the initial study. Concerning the new analysis, steatosis is either present or not present, and should not be subjected to any other arbitrary classifications. Classifying the patients with mild steatosis and the patients with no steatosis into the same group is flawed. Furthermore, since HBsAg seroclearance is a function of time, whether it occurs at an earlier age in patients with steatosis can only be answered by the use of Kaplan-Meier analysis. Multivariate analysis is also needed to determine whether indeed steatosis is a significant factor rather than a co-factor of potentially more important attributes such as obesity, hyperlipidemia, lipid lowering agents, and other metabolic factors.

In fact, spontaneous seroclearance of HBsAg is a rare event in patients with $\mathrm{CHB}$, with an estimated incidence of $0.1-0.8 \%$ annually. This alone makes any studies on HBsAg seroclearance difficult, especially when it usually occurs late in the course of infection. In order to ascertain any definite factors which may contribute to $\mathrm{HBsAg}$ seroclearance, large population studies of unselected $\mathrm{CHB}$ patients are required, and the patients need to be prospectively followed up over a long period of time. It is therefore difficult to draw conclusions based on a case-controlled study and a retrospective cohort study from a single center using a very similar group of patients. As steatosis and metabolic factors are strongly associated with each other, it is also imperative to ensure that these confounding factors are taken into consideration, as they may all have potential effects on the course of HBV infection. Previous large population studies have shown that, even though the presence of metabolic factors is associated with worse liver outcomes in CHB, steatosis was not an independent contributor after controlling for other metabolic components. It is also likely that viral factors such as replication fitness and genotypes, and viral suppression by antiviral therapy, should be predominant factors in HBsAg seroclearance. The presence of low or undetectable HBV DNA would also be a prerequisite factor. In a study of 3,087 CHB patients by Liu et al. [15], 562 patients underwent HBsAg seroclearance after 24,829 person-years of follow-up. Viral load at baseline and its reduction during the follow-up period were the strongest predictors of $\mathrm{HBsAg}$ seroclearance. Interestingly, this study also demonstrated that extreme obesity (BMI $\geq 30 \mathrm{~kg} / \mathrm{m}$ [2]) in men regardless of age was also associated with seroclearance of HBsAg. Whether this is due to underlying steatosis is not known.

In recent years, we have seen an exponential increase in studies examining the use of quantitative HBsAg levels for predicting disease outcome and treatment response [16]. This provides the perfect opportunity to study the kinetics of levels of HBsAg in patients undergoing HBsAg seroclearance. It is likely that, if indeed steatosis does have a favorable effect on HBsAg seroclearance, this will be reflected by a decline in HBsAg levels over time- a trend that should be observed prior to seroclearance. As HBsAg seroclearance is uncommon, measuring HBsAg levels can be an important tool to provide vital clues to either support 
or refute the beneficial effects of steatosis in HBsAg seroclearance.

It is important to stress that, although metabolic factors and steatosis are strongly linked, they may independently have different effects on CHB. The real difficulty is in separating out these factors in order to identify the true effects of steatosis. The evidence thus far demonstrates that metabolic risk factors including obesity are detrimental to the progression of $\mathrm{HBV}$ disease, with increased risk of cirrhosis, HCC, and liver-related mortality (the findings of Liu et al. [15] being the exception). Steatosis, however, appears to be favorable in some instances with less fibrosis and lower viral loads. Chu et al. [13, 14] in their two studies found that steatosis may have a favorable effect on HBsAg seroclearance. This unexpected finding deserves further well-designed studies to confirm and to examine for the possible mechanisms.

Open Access This article is distributed under the terms of the Creative Commons Attribution Noncommercial License which permits any noncommercial use, distribution, and reproduction in any medium, provided the original author(s) and the source are credited.

\section{References}

1. Yuen MF, Yuan HJ, Wong DK, et al. Prognostic determinants for chronic hepatitis B in Asians: therapeutic implications. Gut. 2005;54:1610-1614.

2. Chen CJ, Yang HI, Su J, et al. Risk of hepatocellular carcinoma across a biological gradient of serum hepatitis B virus DNA level. JAMA. 2006;295:65-73.

3. Lim JK, Nguyen MH. The role of hepatic steatosis in chronic hepatitis B infection. Curr Hepatitis Rep. 2011;10:134-141.

4. Kim KH, Shin HJ, Kim K, et al. Hepatitis B virus X protein induces hepatic steatosis via transcriptional activation of SREBP1 and PPARgamma. Gastroenterology. 2007;132:1955-1967.
5. Wong VW, Wong GL, Chu WC, et al. Hepatitis B virus infection and fatty liver in the general population. J Hepatol. 2012;56: 533-540.

6. Yu MW, Shih WL, Lin CL, et al. Body-mass index and progression of hepatitis B: a population-based cohort study in men. J Clin Oncol. 2008;26:5576-5582.

7. Chen CL, Yang HI, Yang WS, et al. Metabolic factors and risk of hepatocellular carcinoma by chronic hepatitis B/C infection: a follow-up study in Taiwan. Gastroenterology. 2008;135: 111-121.

8. Wong GL, Wong VW, Choi PC, et al. Metabolic syndrome increases the risk of liver cirrhosis in chronic hepatitis B. Gut. 2009;58:111-117.

9. Machado MV, Oliveira AG, Cortez-Pinto H. Hepatic steatosis in hepatitis B virus infected patients: meta-analysis of risk factors and comparison with hepatitis C infected patients. J Gastroenterol Hepatol. 2011;26:1361-1367.

10. Shi JP, Fan JG, Wu R, et al. Prevalence and risk factors of hepatic steatosis and its impact on liver injury in Chinese patients with chronic hepatitis B infection. J Gastroenterol Hepatol. 2008; 23:1419-1425.

11. Yun JW, Cho YK, Park JH, et al. Hepatic steatosis and fibrosis in young men with treatment-naive chronic hepatitis B. Liver Int. 2009;29:878-883.

12. Lesmana LA, Lesmana CR, Pakasi LS, Krisnuhoni E. Prevalence of hepatic steatosis in chronic hepatitis B patients and its association with disease severity. Acta Med Indones. 2012;44:35-39.

13. Chu CM, Lin DY, Liaw YF. Does increased body mass index with hepatic steatosis contribute to seroclearance of hepatitis B virus (HBV) surface antigen in chronic $\mathrm{HBV}$ infection? Int $J$ Obes (Lond). 2007;31:871-875.

14. Chu CM, Lin DY, Liaw YF. Clinical and virological characteristics post $\mathrm{HBsAg}$ seroclearance in hepatitis B virus carriers with hepatic steatosis versus those without. Dig Dis Sci. 2012. (Epub ahead of print). doi:10.1007/s10620-012-2343-9.

15. Liu J, Yang HI, Lee MH, et al. Incidence and determinants of spontaneous hepatitis B surface antigen seroclearance: a community-based follow-up study. Gastroenterology. 2010;139: 474-482.

16. Seto WK, Wong DK, Fung J, Hung IF, Fong DY, Yuen JC, Tong $\mathrm{T}$, et al. A large case-control study on the predictability of hepatitis B surface antigen levels three years before hepatitis B surface antigen seroclearance. Hepatology 2012;56:812-819. 